\title{
High energy supercontinuum sources using tapered photonic crystal fibers for multispectral photoacoustic microscopy
}

Magalie Bondu

Christopher Brooks

Christian Jakobsen

Keith Oakes

Peter Morten Moselund

Lasse Leick

Ole Bang

Adrian Podoleanu 


\title{
High energy supercontinuum sources using tapered photonic crystal fibers for multispectral photoacoustic microscopy
}

\author{
Magalie Bondu, ${ }^{\mathrm{a}, \mathrm{b}, \star}$ Christopher Brooks, ${ }^{\mathrm{a}}$ Christian Jakobsen, ${ }^{a}$ Keith Oakes, ${ }^{\mathrm{c}}$ Peter Morten Moselund, ${ }^{\mathrm{a}}$ \\ Lasse Leick, ${ }^{a}$ Ole Bang, ${ }^{\mathrm{a}, \mathrm{d}}$ and Adrian Podoleanu ${ }^{\mathrm{b}}$ \\ ${ }^{a}$ NKT Photonics A/S, Blokken 84, Birkerød 3460, Denmark \\ bUniversity of Kent, Applied Optics Group, School of Physical Science, Ingram Building, Canterbury, Kent CT2 7NH, United Kingdom \\ cElforlight Ltd., 4b Brunel Close, Daventry, Northamptonshire NN11 8RB, United Kingdom \\ dTechnical University of Denmark, DTU Fotonik, Department of Photonics Engineering, Kongens Lyngby 2800, Denmark
}

\begin{abstract}
We demonstrate a record bandwidth high energy supercontinuum source suitable for multispectral photoacoustic microscopy. The source has more than $150 \mathrm{~nJ} / 10 \mathrm{~nm}$ bandwidth over a spectral range of 500 to $1600 \mathrm{~nm}$. This performance is achieved using a carefully designed fiber taper with large-core input for improved power handling and small-core output that provides the desired spectral range of the supercontinuum source. (C) 2016 Society of Photo-Optical Instrumentation Engineers (SPIE) [DOI: 10.1117/1.JBO.21.6.061005]
\end{abstract}

Keywords: supercontinuum; light; energy; photoacoustic.

Paper 150735SSR received Oct. 30, 2015; accepted for publication Dec. 31, 2015; published online Feb. 2, 2016.

\section{Introduction}

Photoacoustic microscopy (PAM) is an absorption-based and depth-resolved imaging technique that combines optical excitation of a zone of interest with ultrasonic detection of the created waves. ${ }^{1}$ A high-pulse energy laser illuminates biological tissue, where molecules absorb the optical energy. A rapid thermoelastic expansion generates broadband, high-frequency ultrasonic (acoustic) waves that are used to reconstruct a depth-resolved map of the optical absorption region. A two-dimensional scanning of the excitation laser allows acquisition of a three-dimensional functional image. Absorption spectra of several main absorbers contained in human tissue are shown in Fig. 1.

For functional bioimaging based on absorption, the window of interest is between 200 and $2000 \mathrm{~nm}$ approximately. Using multiple wavelengths in so-called functional PAM allows both imaging and differentiation of multiple absorbers within a sample. A typical example is the imaging of melanomas, where wavelengths around 584 and $764 \mathrm{~nm}$ can be used to differentiate hemoglobin and melanin and thereby differentiate the melanoma from the surrounding vasculature. ${ }^{9}$

Originally, PAM was demonstrated using single-wavelength lasers as the optical excitation source, such as an Nd:YAG $Q$-switched laser at $1064 \mathrm{~nm}$ or frequency-doubled to $532 \mathrm{~nm}$, or dye lasers. The pulse width is typically 1 to $10 \mathrm{~ns}$ with a pulse repetition rate in the hertz to tens of kilohertz range. ${ }^{10-12}$ Fixed single-wavelength sources made such systems heavily reliant on sample absorption at or near the laser wavelength and are not suitable for multispectral PAM. Optical parametric oscillators (OPOs) have been used for multispectral functional PAM to allow wavelength tunability, reaching a broad tuning range covering 270 to $1300 \mathrm{~nm}^{13}$ However, OPOs have a low pulse repetition rate and are bulky and expensive. Stimulated

*Address all correspondence to: Magalie Bondu, E-mail: mbo@nktphotonics .com
Raman scattering fiber-laser sources have also been used for multispectral functional PAM; however, the accessible wavelengths were limited. ${ }^{14}$

These limitations can be circumvented using a broadband supercontinuum source, which today can be constructed to have over two octaves of bandwidth from about 500 to above $2200 \mathrm{~nm},{ }^{15}$ and can be used in conjunction with optical bandpass filters, such as acousto-optic tunable filters to select the suitable emission wavelength that would allow a fast tunability between different wavelengths (typically tens of kilohertz). Such a strategy would allow multispectral functional PAM capable of interrogating samples containing a wide range of absorbers using a single optical source. However, even state-of-the-art supercontinuum sources do not deliver enough pulse energy to make them suitable for use in multispectral PAM, which requires more than $1.25 \mathrm{~nJ} / \mathrm{nm} .^{16,17}$

Here, we address this issue and present a careful design of a nonlinear fiber for spectral broadening, which is fabricated by tower tapering and combines a large-core input fiber for high-pulse energy handling with a small-core fiber that gives the desired spectral bandwidth. With this nonlinear fiber, we achieve a supercontinuum source with a pulse energy density above $15 \mathrm{~nJ} / \mathrm{nm}$ over a broad bandwidth of 500 to $1600 \mathrm{~nm}$.

\section{Fiber Taper Design}

Our aim is to increase the pulse energy density of the supercontinuum source over a bandwidth as broad as possible, ideally covering at least the 500 to $1000 \mathrm{~nm}$ wavelength range. The pulse energy scales with the pulse width and the peak power. For PAM, the pulse width is typically 1 to 10 ns to enable stress and thermal confinement. ${ }^{1,18}$ Hence, to increase the pulse energy density in a certain wavelength band of the supercontinuum spectrum, a pump laser with a pulse width close to the maximum

$1083-3668 / 2016 / \$ 25.00$ @ 2016 SPIE 


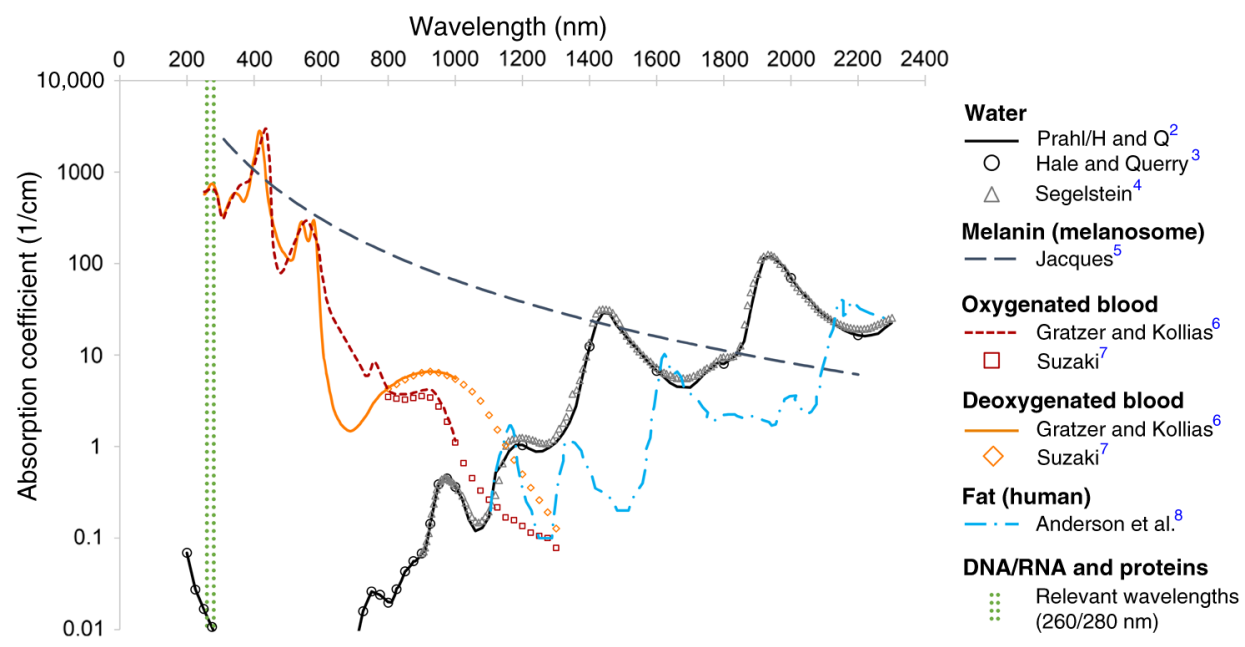

Fig. 1 Absorption coefficients of several main absorbers contained in human tissue. The graphs for water have been drawn by adapting data from Refs. 2-4. The graph for the melanin (melanosoma) was inferred using Eq. (8) in Ref. 5. Oxygenated and deoxygenated blood graphs have been obtained by adapting data from Refs. 6 and 7 . The graph for human fat has been drawn using data from Ref. 8. 260 and $280 \mathrm{~nm}$ are the relevant wavelengths used for DNA, RNA base identification, and DNA/proteins ratio measurement.

$10 \mathrm{~ns}$ should be used, and the pulse peak power in that band must be increased.

Supercontinuum sources typically comprise a pump laser coupled into a highly nonlinear fiber. ${ }^{15}$ To increase the peak power within a specific wavelength range one can (1) increase the pump peak power, which will both increase the power and change the spectral profile of the supercontinuum, or (2) change the fiber design to spectrally shape the supercontinuum and move power from a nonimportant wavelength range into the desired wavelength range. ${ }^{19,20}$ We report on a method using both solutions.

Most common commercial supercontinuum sources use a microstructured photonic crystal fiber (PCF) with a small-core diameter, both to move the zero-dispersion wavelength (ZDW) below the pump laser wavelength of $1064 \mathrm{~nm}^{21}$ and to achieve a certain blue edge, typically below $500 \mathrm{~nm} .{ }^{22}$ Standard supercontinuum PCFs have a guiding core diameter of $<5 \mu$ m surrounded by a microstructure consisting of air holes of diameter $d$ with a relative hole diameter, or air-hole diameter to pitch ratio $(d / \Lambda)$, of around 0.5 (e.g., the SC-5.0-1040 from NKT Photonics), and generate a broad spectrum with a blue edge around $475 \mathrm{~nm}$ and a red edge above $2300 \mathrm{~nm}^{20}$

The small core increases the effective nonlinearity of the fiber and thus the efficiency of the supercontinuum generation (SCG), but it also limits the power-handling capability. Since the maximum optical pulse peak power a fiber can handle is linked to the energy per surface area at a given pulse width, it would be desirable to use a larger core fiber to allow higher energy pulses to be coupled into the fiber. However, since the nonlinearity is inversely proportional to the effective mode area, simply increasing the core diameter leads to less efficient SCG, in particular if it moves the ZDW above the pump wavelength so that the pump is in the normal dispersion regime. ${ }^{23}$ More importantly, by increasing the core diameter, the blue edge of the spectrum is typically shifted to longer wavelengths, ${ }^{20,22,24}$ reducing coverage of the PAM window.

Our solution is to use a carefully designed PCF taper, which connects an input and output PCF, each with their carefully chosen designs: a large-core fiber that can handle high-pulse energy at the input and a standard highly nonlinear small-core PCF at the output that produces the desired blue edge of the output spectrum. Tapered fibers were used early on for $\mathrm{SCG}^{25}$ and have been thoroughly studied in terms of how they shift the dispersion wavelength and supercontinuum spectral edges (see Refs. 19 and 20 for recent reviews).

The simplest tower tapering method only changes the drawing speed in order to make the taper and thereby, to a good approximation, maintains the relative hole diameter $d / \Lambda$ while reducing the hole-to-hole pitch $\Lambda$. In this case, there is an optimum pitch that gives the shortest blue edge determined by group-velocity matching to the red edge. ${ }^{20,24}$ For a relative hole diameter of 0.52 , the optimum pitch is, for instance, $2.6 \mu \mathrm{m}$, which results in a blue edge of around $479 \mathrm{~nm}$ depending on the infrared loss edge. ${ }^{24}$ Interestingly, this optimum pitch is typically the one for which the second ZDW crosses the infrared loss edge of the fiber. ${ }^{24}$ The amount of power in the blue edge is determined by so-called group-acceleration matching and decreases with the steepness of the taper (i.e., long tapers have more power closer to the blue spectral edge). ${ }^{24,26}$

While most of the focus in fiber tapering has been on expanding spectral coverage at the blue edge, it should be mentioned that theoretical work has also been done on designing special taper profiles that allow to manipulate the red edge in terms of optimizing the Raman-induced soliton self-frequency shift. ${ }^{27}$

When tapering PCFs in particular, a more complicated strategy of draw tower tapering is employed, where both the draw speed and the pressure on the air holes are controlled. Such a strategy allows optimum control of the air hole microstructure along the taper. This was used to make a supercontinuum PCF taper with a single-mode input $(d / \Lambda=0.52, \Lambda=3.3 \mu \mathrm{m})$ and a large relative hole diameter at the output $(d / \Lambda=0.85$, $\Lambda=2.2 \mu \mathrm{m}$ ) for obtaining a deep-blue supercontinuum with a blue edge at $375 \mathrm{~nm}^{28}$

In view of these results and general design rules, we choose here simple tower tapering where only the drawing speed is varied to change the core diameter with a fixed relative hole diameter. This gives the best taper reproducibility and is sufficient here since we have chosen two input and output PCF designs 
that have the same relative hole diameter of 0.52 . To avoid too much spectral broadening in the tapered section connecting our two fiber sections, we use rather short 0.5 to $1 \mathrm{~m}$ tapered sections. The fiber output section is similar to a standard supercontinuum fiber with a $5 \mu \mathrm{m}$ core diameter and the input section is a larger core fiber with a core diameter above $10 \mu \mathrm{m}$ for increased power handling. The ZDW of the input section of the fiber is above $1170 \mathrm{~nm}$, and thus we are pumping $100 \mathrm{~nm}$ into the normal dispersion regime of a large-core fiber with long pulses. Significant spectral broadening is therefore not expected before the optical pulse reaches near the end of the tapered section where the ZDW crosses the pump wavelength of $1064 \mathrm{~nm}$.

\section{Experimental Setup and Specific Fibers}

The experimental setup is shown in Fig. 2. A 1064-nm Elforlight laser (Model FQS-400-1-Y-1064) emitting pulses with a duration of $4 \mathrm{~ns}$, a repetition rate of $1 \mathrm{kHz}$, and pulse energies of up to $500 \mu \mathrm{J}$ is used as pump laser. A half-wave plate (HWP) and a polarizing beam splitter (PBS) allow for continuous adjustment of the laser power, while an aspheric lens (AL) is used to couple the pump into the PCF. The AL focal length is optimized for each PCF in order to maximize the coupling efficiency. The PCF input end is fixed to a NanoMax three-axis precision translation stage, and the angle of the input beam with respect to the $\mathrm{AL}$ can be adjusted with two steering mirrors in order to optimize coupling, which for this work was typically around $60 \%$, so that the total output energy of the supercontinuum is typically around $20 \%$ to $30 \%$ of the pump laser. An integrating sphere is used to collect the output signal and relay it to an optical spectrum analyzer (OSA) via a large-core step-index fiber. Two separate OSAs are used in order to record the full spectrum, one with a scanning range from 350 to $1750 \mathrm{~nm}$ (Yokogama, Ando AQ6315) and a second one covering a range from 1200 to $2400 \mathrm{~nm}$ (Yokogama, AQ6375). A broadband power meter (Ophir Photonics, 3A-QUAD) is used to measure the total optical power in the supercontinuum. The supercontinuum spectrum is then calibrated using all measurements and plotted in terms of energy density in $\mathrm{nJ} / \mathrm{nm}$.

We tested five different PCFs drawn by NKT Photonics, four of which were specially designed for this study with an approximately fixed relative hole diameter of 0.52 . There are three straight PCFs with core diameters of 5 (SC-5.0-1040), 9 (PCF-9), and $10 \mu \mathrm{m}$ (PCF-10) and two PCF tapers with an input core diameter of $\sim 10 \mu \mathrm{m}$ tapered down to $5 \mu \mathrm{m}$ over a length of 0.5 to $1 \mathrm{~m}$ (tapers 10.3-5 and 10.7-5). The specific longitudinal profile and the input and output end facets of the tapered fibers are given in Fig. 3.

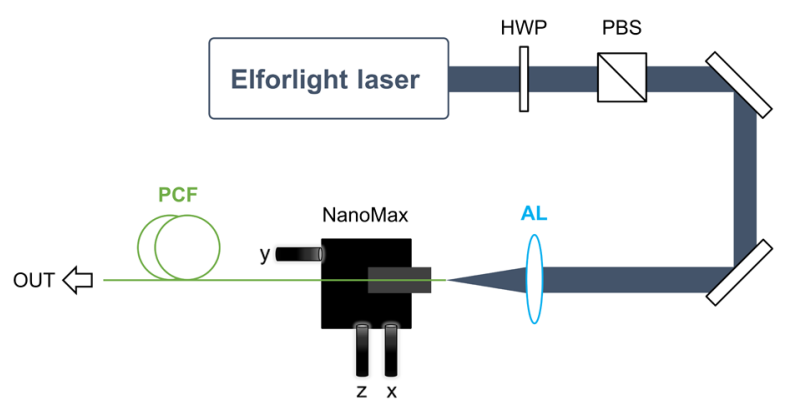

Fig. 2 Experimental setup.

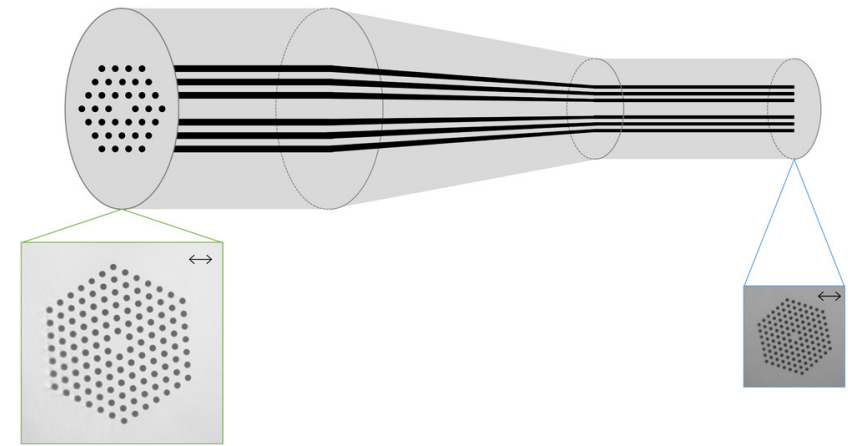

Fig. 3 Longitudinal profile of the tapered fibers with microscope images of the end facets for taper 10.7-5 (black arrow represents $10 \mu \mathrm{m}$ ). The length of the straight input section is $\sim 2 \mathrm{~m}$, and the total fiber length is $\sim 15 \mathrm{~m}$. The length of the tapered section is about $1 \mathrm{~m}$ for taper 10.7-5 and 0.5 $\mathrm{m}$ for taper 10.3-5.

\section{Experimental Results}

For each of the five PCFs, we measure the pump pulse energy it can transmit before damage occurs and the supercontinuum spectrum at a pump pulse energy just below the damage threshold. Figure 4 shows the damage threshold for several PCF input end core diameters and illustrates the linear dependence of the damage threshold with the core area at the input end of the fiber. More importantly, we see no difference between the straight fibers and tapered fibers with similar input core diameter. Hence, the damage threshold of the tapered fibers is that of the input fiber; that is, no damage occurs within the tapered and straight sections.

We conclude that the input core diameter is, as we thought, the relevant parameter for determining the maximum permissible energy that can be coupled into a PCF.

Figure 5 shows the supercontinuum spectrum generated by each of the standard PCFs (SC-5.0-1040, PCF-9, and PCF-10) and the taper designated as 10.7-5. The optical spectra have been recorded using an input energy corresponding to $\sim 70 \%$ to $90 \%$ of the damage threshold.

Table 1 lists the core diameter, the total supercontinuum output pulse energy, the visible pulse energy, and the theoretical and experimental blue edges. The visible energy is measured by filtering out the light above $850 \mathrm{~nm}$ and the blue edge is evaluated at the $2 \mathrm{~nJ} / \mathrm{nm}$ level.

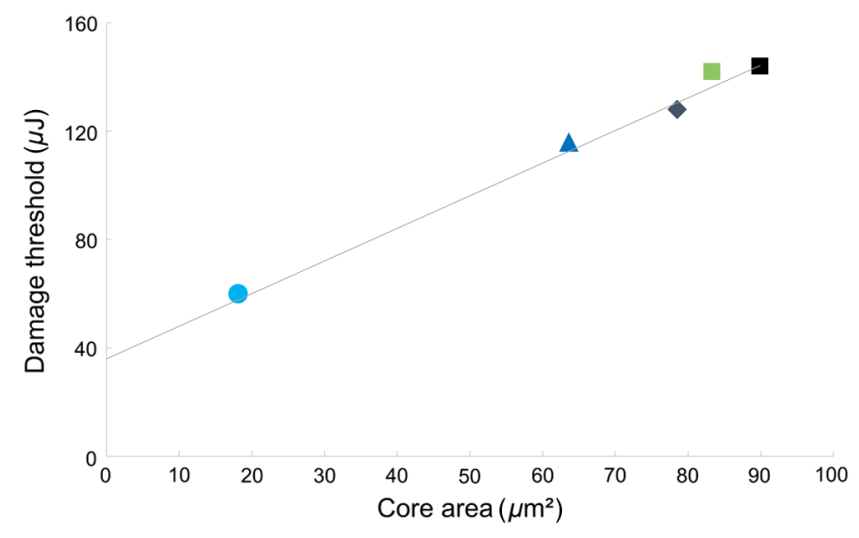

Fig. 4 Damage threshold versus core area for the PCFs used in these tests. Light blue circle: SC-5.0-1040. Blue triangle: PCF-9. Dark diamond: PCF-10. Green square: tapered 10.3-5. Black square: tapered 10.7-5. 


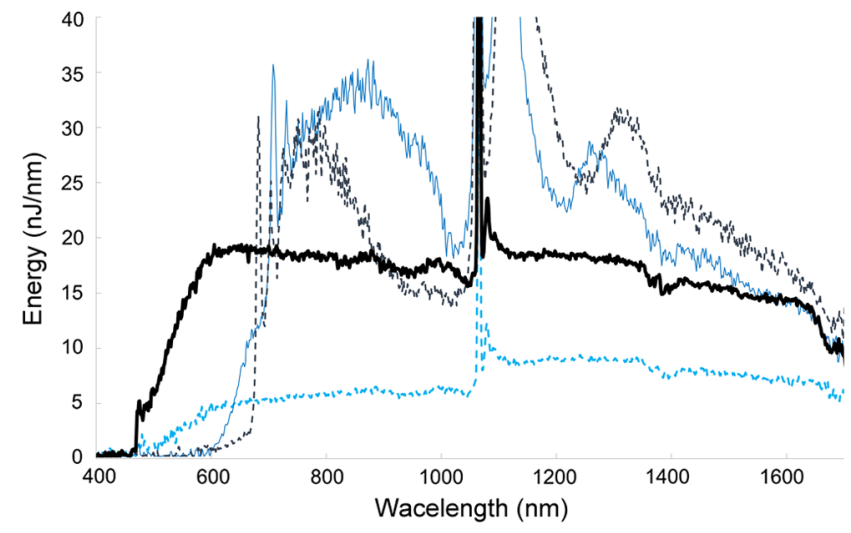

Fig. 5 Supercontinuum output spectra from the various PCF designs tested. Blue-dashed line: SC-5.0-1040. Blue solid line: PCF-9. Darkdashed line: PCF-10. Thick black solid line: tapered 10.7-5.

Table 1 Characteristics of four PCFs and their generated spectra. The relative hole diameter and the total fiber length are fixed at $\sim 0.52$ and $15 \mathrm{~m}$, respectively.

\begin{tabular}{lcccc} 
& $\begin{array}{c}\text { SC-5.0- } \\
1040\end{array}$ & PCF-9 & PCF-10 & $\begin{array}{c}\text { Tapered } \\
10.7-5\end{array}$ \\
\hline Core diameter $(\mu \mathrm{m})$ & 5 & 9 & 10 & $10.7-5$ \\
Total output energy $(\mu \mathrm{J})$ & 10 & 29.5 & 30 & 22 \\
Visible output energy $(\mu \mathrm{J})$ & 1.7 & 5.4 & 4.6 & 6 \\
Experimental blue edge $(\mathrm{nm})$ & $\sim 475$ & $\sim 600$ & $\sim 675$ & $\sim 475$ \\
Theoretical blue edge $(\mathrm{nm})^{\mathrm{a}}$ & 496 & 582 & 594 & 496 \\
\hline
\end{tabular}

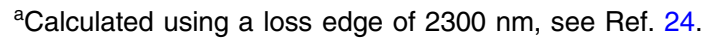

The spectra shown in Fig. 5 confirm that the tapered fiber indeed provides a much higher pulse energy density while simultaneously emitting a supercontinuum with a blue edge that remains at $475 \mathrm{~nm}$. We also see that the large-core straight fibers perform equally as well as the tapered fiber in term of power handling; however, the blue edge is too far shifted toward longer wavelengths to be of any use for functional multispectral PAM. Table 1 shows that the available visible pulse energy from the tapered fiber is improved by more than a factor of 3 over the $5-\mu \mathrm{m}$ core standard fiber, and the pulse energy density exceeds $15 \mathrm{~nJ} / \mathrm{nm}$ within the 500 to $1600 \mathrm{~nm}$ wavelength range.

\section{Conclusion}

We have demonstrated a supercontinuum source especially suitable for functional multispectral PAM. The source exhibits a significantly enhanced output pulse energy within a broad bandwidth where a wide range of human tissue components manifest high absorption. The supercontinuum source uses a carefully designed tapered PCF, which combines the enhanced power handling of a large-core input fiber with the high nonlinearity and spectral broadening performance of a small-core PCF. The taper design allows higher supercontinuum output pulse energy without shifting of the blue edge of the spectrum from $475 \mathrm{~nm}$. The obtained supercontinuum source has a pulse energy density above $15 \mathrm{~nJ} / \mathrm{nm}$ over a record $1100 \mathrm{~nm}$ bandwidth, covering 500 to $1600 \mathrm{~nm}$. This source, when combined with optical bandpass filters, can serve functional multispectral PAM applications.

The supercontinuum output energy can be further scaled up by increasing the input core diameter and then use a pump source with a higher peak power. The pulse width cannot be increased much from the $4 \mathrm{~ns}$ values used here, due to the inherent restrictions in PAM, which requires stress and thermal confinement.

\section{Acknowledgments}

The research is supported by the Marie Curie action of the European Research Commission under the projects UBAPHODESA (FP7 FP7-PEOPLE 607627) and FAMOS (FP7 ICT 317744). A. Podoleanu is also supported by the NIHR Biomedical Research Centre at Moorfields Eye Hospital NHS Foundation Trust and the UCL Institute of Ophthalmology.

\section{References}

1. J. Yao and L. V. Wang, "Photoacoustic microscopy," Laser Photonics Rev. 7(5), 758-778 (2013).

2. G. M. Hale and M. R. Querry, "Optical constants of water in the $200 \mathrm{~nm}$ to $200 \mu \mathrm{m}$ wavelength region," Appl. Opt. 12(3), 555-563 (1973). (compiled and posted on http://omlc.org/spectra/water/data/hale73.dat by $\mathrm{S}$. Prahl).

3. G. M. Hale and M. R. Querry, "Optical constants of water in the $200 \mathrm{~nm}$ to $200 \mu \mathrm{m}$ wavelength region," Appl. Opt. 12(3), 555-563 (1973).

4. D. J. Segelstein, "The complex refractive index of water," Master of Science Thesis, University of Missouri, Kansas City (1981).

5. S. L. Jacques, "Optical properties of biological tissues: a review," Phys. Med. Biol. 58, R37 (2013).

6. W. B. Gratzer and N. Kollias, Personal communication from Gratzer, Med. Res. Council Labs, Holly Hill, London, and N Kollias, Wellman Laboratories, Harvard Medical School, Boston (1999) (compiled and posted on http://omlc.org/spectra/hemoglobin/ by S. A. Prahl).

7. H. Suzaki, "Noninvasive measurement of total hemoglobin and hemoglobin derivatives using multiwavelength pulse spectroscopy-In vitro study with a mock circulatory system," in Proc. of the 28th IEEE EMBS Annual Int. Conf., New York City, August 30-September 3, 2006 (2006) (compiled and posted on http://omlc.org/news/feb15/ generic_optics/bloodSuzaki.dat by S. Jacques).

8. R. R. Anderson et al., "Selective photothermolysis of lipid-rich tissues: a free electron laser study," Laser Surg. Med. 38, 913-919 (2006) (compiled and posted on http://omlc.org/news/feb15/generic_optics/ AndersonFat.m by S. Jacques).

9. H. F. Zhang et al., "Functional photoacoustic microscopy for highresolution and noninvasive in vivo imaging," Nat. Biotechnol. 24(7), 848-851 (2006).

10. K. Maslov, G. Stoica, and L. V. Wang, "In vivo dark-field reflectionmode photoacoustic microscopy," Opt. Lett. 30(6), 625-627 (2005).

11. T. Liu et al., "Near-infrared light photoacoustic ophthalmoscopy," Biomed. Opt. Express 3(4), 792-799 (2012).

12. W. Liu et al., "In vivo corneal neovascularization imaging by opticalresolution photoacoustic microscopy," Photoacoustics 2, 81-86 (2014).

13. R. Cao et al., "Multispectral photoacoustic microscopy based on an optical-acoustic objective," Photoacoustics 3, 55-59 (2015).

14. P. Hajireza, A. Forbrich, and R. Zemp, "In-vivo functional opticalresolution photoacoustic microscopy with stimulated Raman scattering fiber-laser source," Biomed. Opt. Express 5(2), 539-546 (2014).

15. J. M. Dudley, G. Genty, and S. Coen, "Supercontinuum generation in photonic crystal fiber," Rev. Mod. Phys. 78(4), 1135 (2006).

16. Y. N. Billeh, M. Liu, and T. Buma, "Spectroscopic photoacoustic microscopy using a photonic crystal fiber supercontinuum source," Opt. Express 18(18), 18519 (2010).

17. C. Lee et al., "In vitro photoacoustic measurement of hemoglobin oxygen saturation using a single pulsed broadband supercontinuum laser source," Appl. Opt. 53(18), 3884 (2014). 
18. M. Xu and L. V. Wang, "Photoacoustic imaging in biomedicine," Rev. Sci. Instrum. 77, 041101 (2006).

19. J. C. Travers, "Blue extension of optical fibre supercontinuum generation," J. Opt. 12, 113001 (2010).

20. U. Møller et al., "Optimum PCF tapers for blue-enhanced supercontinuum sources," Opt. Fiber Technol. 18, 304 (2012).

21. J. K. Ranka, R. S. Windeler, and A. J. Stentz, "Visible continuum generation in air-silica microstructure optical fibers with anomalous dispersion at $800 \mathrm{~nm}, "$ Opt. Lett. 25(1), 25 (2000).

22. J. M. Stone and J. C. Knight, "Visibly "white' light generation in uniform photonic crystal fiber using a microchip laser," Opt. Express 16(4), 2670 (2008).

23. U. Møller and O. Bang, "Intensity noise in normal-pumped picoseconds supercontinuum generation, where higher-order Raman lines cross into the anomalous dispersion regime," Electron. Lett. 49(1), 63 (2013)

24. S. T. Sørensen et al., "Deep-blue supercontinnum sources with optimum taper profiles-verification of GAM," Opt. Express 20(10), 10635 (2012).

25. T. A. Birks, W. J. Wadsworth, and P. St. J. Russell, "Supercontinuum generation in tapered fibers," Opt. Lett. 25(19), 1415 (2000).

26. S. T. Sørensen et al., "Optimum fiber tapers for increasing the power in the blue-edge of a supercontinuum-group-acceleration matching," Opt. Lett. 36(6), 816 (2011).

27. A. Judge et al., "Optimization of the soliton self-frequency shift in a tapered photonic crystal fiber," J. Opt. Soc. Am. B 26(11), 2064 (2009).

28. S. T. Sørensen et al., "Single-mode pumped high air-fill fraction photonic crystal fiber taper for high-power deep-blue supercontinuum sources," Opt. Lett. 39(4), 1097 (2014).

Magalie Bondu is a Marie Curie early stage $\mathrm{PhD}$ student (UBAPHODESA, 607627) at NKT Photonics A/S and the University of Kent. She received her BS and MS degrees in laser, optics, and matter from the Institute of Optics Graduate School in 2012 and 2014 , respectively. Her PhD project focuses on creating new supercontinuum designs suitable for photoacoustic microscopy and multimodal imaging using a single broadband source.

Christopher Brooks is a junior researcher with the RP4 Group at ELI Beamlines in Dolní Břežany, Czech Republic, where he focuses on the implementation of high harmonic generation with VUV and soft $x$-ray ellipsometry for materials science research. Prior to this, he worked as a research engineer at NKT Photonics. He has worked extensively with high-peak-power, high-pulse-energy, fiber-based sources for remote sensing and medical applications. He coauthored several awarded patents and peer-reviewed journal articles.
Christian Jakobsen is a development engineer at NKT Photonics with main activities in developing optical fibers and fiber manufacturing processes. He has a PhD in optical physics and has been active in research and development within manufacturing of optical fibers for the past 20 years. He is inventor and coinventor of a number of patents and author and coauthor of a number of papers in peer-reviewed journals.

Keith Oakes is a founding director of Elforlight Ltd. His role is focused on design and development of nanosecond DPSS lasers and OPOs. $\mathrm{He}$ graduated from Hull University in 1980 with BSc joint honors in physics and geology. He has spent 35 years working in laser research, development, and design.

Peter Morten Moselund has worked for nine years with pulsed fiber lasers and supercontinuum generation. For the last four years, he has worked mainly on mid-IR supercontinuum generation as the technical manager of NKT Photonics activities in this field. He authored or coauthored more than 35 refereed articles, nearly all within the field of supercontinuum generation and its applications (369 citations, h-index 12). In addition, he is the inventor or coinventor of two patent families.

Lasse Leick is heading the external projects and IPR Group at NKT Photonics. He has a PhD in components for optical telecommunications, and since 2006 he has been working on ultrafast pulsed lasers and supercontinuum sources. He has authored or coauthored more than 30 refereed articles (371 citations, h-index 11). In 2012, he received the Danish National Advanced Technology Foundation's Pasteur Prize, which is given to the project leader of the year.

Ole Bang is a professor at the Technical University of Denmark and Fellow of OSA. He heads the Fiber Sensors \& Supercontinuum Group, who develops photonic crystal fibers for sensing and supercontinuum lasers and use them for applications in imaging and spectroscopy. He has published 180 journal papers with 6040 ISI citations and ISI h-index 44 . He holds several patents within supercontinuum lasers and polymer optical fiber sensors and is co-founder of SHUTE Sensing Technologies.

Adrian Podoleanu is a professor of biomedical optics at the University of Kent, UK, and he is a fellow of OSA, SPIE, and IOP. He leads the research of the Applied Optics Group, oriented on high resolution noninvasive optical imaging. The awards he received are Royal Society Wolfson Research Merit Award 2015; ERC Advanced Research Fellowship, 2010 to 2015; Ambassador's Diploma, Embassy of Romania in UK, 2009; Leverhulme Research Fellowship, 2004 to 2006; and the Romanian Academy Constantin Miculescu Prize, 1984. 